\title{
Erratum
}

\section{Van Kampen's expansion approach in an opinion formation model}

M.S. de la Lama ${ }^{1, a}$, I.G. Szendro ${ }^{1, b}$, J.R. Iglesias ${ }^{2,3, c}$, and H.S. Wio ${ }^{1, d}$

1 Instituto de Física, Universidad de Cantabria and CSIC, 390005-Santander, Spain

2 Instituto de Física, Universidade Federal de Rio Grande do Sul, 91501-970 Porto Alegre, Brazil

3 Programa de Pós Graduação em Economia, Universidade Federal de Rio Grande do Sul, Av. João Pessoa 52, $90040-000$ Porto Alegre, Brazil

Eur. Phys. J. B 51, 435 (2006)

Received 17 July 2007

Published online 1st August 2007 - (c) EDP Sciences, Società Italiana di Fisica, Springer-Verlag 2007

In Eur. Phys. J. B 51, 435 (2006), equation (2) that reads

$$
\begin{aligned}
\frac{\partial}{\partial t} P\left(N_{A}, N_{B}, t\right)= & \alpha_{1}\left(N_{A}+1\right) P\left(N_{A}+1, N_{B}, t\right)+\alpha_{3}\left(N_{B}+1\right) P\left(N_{A}, N_{B}+1, t\right) \\
+ & \alpha_{2}\left(N-N_{A}-N_{B}+1\right) P\left(N_{A}-1, N_{B}, t\right) \\
+ & \alpha_{4}\left(N-N_{A}-N_{B}+1\right) P\left(N_{A}, N_{B}-1, t\right) \\
+ & \frac{\beta_{1}}{N}\left(N_{A}-1\right)\left(N-N_{A}-N_{B}+1\right) P\left(N_{A}-1, N_{B}, t\right) \\
+ & \frac{\beta_{2}}{N}\left(N_{B}-1\right)\left(N-N_{A}-N_{B}+1\right) P\left(N_{A}, N_{B}-1, t\right) \\
& -\left[\alpha_{1} N_{A}+\alpha_{3} N_{B}+\alpha_{2}\left(N-N_{A}-N_{B}\right)+\alpha_{4}\left(N-N_{A}-N_{B}+1\right)\right] P\left(N_{A}, N_{B}, t\right),
\end{aligned}
$$

was erroneously typed. It should be corrected to

$$
\begin{aligned}
\frac{\partial}{\partial t} P\left(N_{A}, N_{B}, t\right)= & \alpha_{1}\left(N_{A}+1\right) P\left(N_{A}+1, N_{B}, t\right)+\alpha_{3}\left(N_{B}+1\right) P\left(N_{A}, N_{B}+1, t\right) \\
+ & \alpha_{2}\left(N-N_{A}-N_{B}+1\right) P\left(N_{A}-1, N_{B}, t\right) \\
+ & \alpha_{4}\left(N-N_{A}-N_{B}+1\right) P\left(N_{A}, N_{B}-1, t\right) \\
+ & \frac{\beta_{1}}{N}\left(N_{A}-1\right)\left(N-N_{A}-N_{B}+1\right) P\left(N_{A}-1, N_{B}, t\right) \\
+ & \frac{\beta_{2}}{N}\left(N_{B}-1\right)\left(N-N_{A}-N_{B}+1\right) P\left(N_{A}, N_{B}-1, t\right) \\
- & {\left[\alpha_{1} N_{A}+\alpha_{3} N_{B}+\left(\alpha_{2}+\alpha_{4}\right)\left(N-N_{A}-N_{B}\right)\right.} \\
& \left.\quad+\left(\frac{\beta_{1} N_{A}+\beta_{2} N_{B}}{N}\right)\left(N-N_{A}-N_{B}\right)\right] P\left(N_{A}, N_{B}, t\right) .
\end{aligned}
$$

The rest of the equations for orders $\Omega^{1 / 2}$ and $\Omega^{0}$, as well as the ones corresponding to the Fokker-Planck equation and its moments, are correct.

\footnotetext{
a e-mail: msanchez@ifca.unican.es

b e-mail: szendro@ifca.unican.es

c e-mail: iglesias@if.ufrgs.br

d e-mail: wio@ifca.unican.es
} 